\title{
Overview of the lithium problem in metal-poor stars and new results on ${ }^{6} \mathbf{L i}$
}

\author{
R. Cayrel ${ }^{a *}$, M. Steffen ${ }^{b}$, P. Bonifacio ${ }^{a c d}$, H.-G. Ludwig ${ }^{a c}$ and E. Caffau ${ }^{a}$ \\ ${ }^{a}$ GEPI, Observatoire de Paris, CNRS, Université Paris Diderot,61 av. de l'Observatoire 75014 \\ Paris, France \\ ${ }^{b}$ Astrophysikalisches Institut Potsdam, An der Sternwarte 16, Potsdam, Germany \\ ${ }^{c}$ CIFIST Marie Curie Excellence Team \\ ${ }^{d}$ Istituto Nazionale di Astrofisica, Osservatorio Astronomico di Trieste, Via G.B. Tiepolo 11, \\ 34143 Trieste, Italy \\ E-mail: roger.cayrel@obspm.fr
}

Two problems are discussed here. The first one is the 0.4 dex discrepancy between the ${ }^{7} \mathrm{Li}$ abundance derived from the spectra of metal-poor halo stars on the one hand, and from Big Bang nucleosynthesis, based on the cosmological parameters constrained by the Wilkinson Microwave Anisotropy Probe (WMAP) measurements, on the other hand. Lithium, indeed, can be depleted in the convection zone of unevolved stars, by a combination of diffusion and slow mixing with the hotter layers below the convection zone, where ${ }^{7} \mathrm{Li}$ is destroyed by the ${ }^{7} \mathrm{Li}(\mathrm{p}, \alpha){ }^{4} \mathrm{He}$ reaction. The understanding of the hydrodynamics of this crucial zone near the bottom of the convective envelope in dwarfs or turn-off stars of solar metallicity has recently made enormous progress with the inclusion of internal gravity waves. However, similar work for metal-poor stars is still lacking. So in spite of several investigations claiming to have partly explained the above mentioned ${ }^{7} \mathrm{Li}$ discrepancy, it is not yet clear whether or not the depletion occurring in the metal-poor stars themselves is adequate to produce a ${ }^{7} \mathrm{Li}$ plateau. The second problem concerns the difficulties met in accounting for the large amount of ${ }^{6} \mathrm{Li}$ recently found in metal-poor halo stars (Asplund et al. 2006 [15]). It has already been suggested (Cayrel et al. 2007 [24]) that the convection-related asymmetry of the ${ }^{7} \mathrm{Li}$ line could mimic the signal attributed so far to the weak blend of ${ }^{6} \mathrm{Li}$ in the red wing of the ${ }^{7} \mathrm{Li}$ line. However, this suggestion was only based on a hydrodynamical simulation for a single set of atmospheric parameters, representing the halo turn-off star HD 74000. Now the theoretical line asymmetry has been computed for an extended range in effective temperature, gravity and metallicity, covering the stars of the Asplund et al. sample. The computed asymmetry is about two per cent, a value which is also the mean of the ${ }^{6} \mathrm{Li} /{ }^{7} \mathrm{Li}$ ratio of the full sample determined with symmetric profiles by Asplund et al. 2006. Our conclusion is that these observations can be reinterpreted in terms of intrinsic line asymmetry, plus the amount of ${ }^{6} \mathrm{Li} /{ }^{7} \mathrm{Li}$ given by Asplund et al. reduced by 0.02 . This drastically reduces the number of certain ${ }^{6} \mathrm{Li}$ detections, from 9 to 1 or 2 .

10th Symposium on Nuclei in the Cosmos

July 27 - August 12008

Mackinac Island, Michigan, USA

${ }^{*}$ Speaker. 


\section{The WMAP / Spite plateau discrepancy}

The Big Bang nucleosynthesis leads now to very accurate predictions of the primordial abundances, with the cosmological parameter $\eta=N$ (baryons) $/ N$ (photons) constrained by the observations of WMAP. On the usual scale of abundances by number of atoms per $10^{12}$ hydrogen atoms, the logarithmic abundance of ${ }^{7} \mathrm{Li}$ is $\log n(\mathrm{Li})=2.64 \pm 0.04$ (Spergel et al. 2007 [1]), against a logaritmic abundance of $2.22 \pm 0.08$ for the Spite plateau (Charbonnel \& Primas 2005 [2]). The first idea which comes to mind is that the nuclear fragility of ${ }^{7} \mathrm{Li}$ can deplete the element by the same mechanism which has depleted this nuclide in the Sun by about 2 dex in 4.6 Gyr. The main difficulty with this scheme is that the Spite plateau is both flat and shows only a very small scatter. It is a priori difficult to imagine a mechanism leading to a constant depletion over two decades in metallicity and almost $1000 \mathrm{~K}$ in effective temperature. The problem of the small scatter was also serious as long as effects of stellar rotation were believed to be a main ingredient of the depletion mechanism. The inital distribution of stellar rotation induces without doubt a statistical scatter. We shall come back to this mechanism in subsection 1.3.

Other possible mechanisms have been proposed. In section 1.1 we shortly discuss the proposal that the depletion of the Spite plateau is due to a massive astration of the cosmic matter before the birth of the galactic halo stars (Piau et al. 2006 [3]). In section 1.2 we quote proposals using supersymmetric particles, very briefly, because the subject is considered elsewhere in this volume. In section 1.3 we discuss in detail the diffusion - slow mixing - nuclear burning mechanism, now widely studied in dwarfs of solar metallicity.

\subsection{A major astration of cosmic matter before the formation of halo stars}

Piau et al. 2006 [3] have proposed that about half of the mass of the cosmic matter from which the halo stars formed was already processed by pregalactic supernovae $(\mathrm{SNe})$ of masses between 10 and $40 M_{\odot}$. Although this scenario has some interesting features concerning, for example, the high depletion of $\mathrm{Li}$ in the extremely iron poor, C-enriched star HE 1327-2326, the level of astration invoked seems incompatible with the expected resulting metallicity on the part of the plateau below $[\mathrm{Fe} / \mathrm{H}]=-2.0$ (Prantzos 2006, NIC-IX [4]).

\subsection{Supersymmetric particles}

If they exist, supersymmetric particles may have played a role, both in depleting ${ }^{7} \mathrm{Li}$ and in producing ${ }^{6} \mathrm{Li}$ during the Big Bang, winning on the two sides. As the topic has been developed elsewhere in this volume, I will just mention here a few references dealing with this topic: Jedamzik 2004 [5], Pospelov 2007 [7], Cumberbatch et al. 2007 [8], and Kusakabe et al. 2007 [9].

\subsection{Depletion by the diffusion - slow mixing - nuclear burning mechanism}

Atmospheres of low mass unevolved stars are rapidly mixed by convection and a small amount of convective overshooting. Below the convection zone, the situation is rather complex. Heavy ions settle down very slowly, but if a new ionization stage is reached, radiative support by continuous plus line absorption can block this motion. Under the impulse of Georges Michaud (see the Meeting in Honor of Georges Michaud 2005 [10]) an enormous amount of work has been done in this field. The situation is complicated by the existence of hydrodynamical effects which tends to modify this 
vertical stratification and by the existence of meridional circulation. Initially, turbulence generated by differential rotation was considered to be the main cause of mixing, inhibiting to some degree the effects of diffusive sedimentation. The presence of hydrodynamical turbulence which tends to scramble the effects of diffusion, also enables the depletion of fragile nuclides which are destroyed at higher temperatures some depth below the bottom of the convection zone. In that case, the hydrodynamical turbulence creates a slow mixing between the convection zone and the burning region below. Very clearly, this is the case for Sun, in which the photospheric ${ }^{7} \mathrm{Li}$ is depleted by more than a factor 100 from an ISM initial value of 3.3. Korn et al. 2007 [11] have constrained the role of diffusion by comparing the abundances of a few elements in unevolved stars and evolved stars in the globular cluster NGC 6397. They derive a depletion of ${ }^{7} \mathrm{Li}$ by about 0.25 dex using a stellar model of $\mathrm{O}$. Richard (see [10]). This value depends upon the difference of effective temperature between the unevolved stars and the evolved stars, and upon the empirical model of Richard, done before the theoretical work of Charbonnel \& Talon 2008 [14]. The latter approach has been very successful in explaining a large body of observations able to constrain the theory. First the evolution with time can be checked by observing stars in clusters of various ages, from the Pleiades to M67. Then the Boesgaard-Tripicco dip [12] poses a severe challenge to the theory, which was resolved only very recently [14]. The simultaneous observation of the three fragile nuclides ${ }^{7} \mathrm{Li},{ }^{7} \mathrm{Be}$, and ${ }^{9} \mathrm{~B}$ in field stars (Boeesgaard et al. 1998 [13]) supplies direct evidence that the depletion is linked to the physical properties of the nuclei, and reaches levels unattainable by diffusion only. This body of observations has allowed to put the theory of loss of angular momentum and of chemical mixing in cool stars on a much better footing than before. A key ingredient has been to take into consideration internal gravity waves as well as the effects of rotation (meridional circulation and shear turbulence) [14]. Unfortunately the low-metallicity stars of the galactic halo have not yet been investigated in as much detail as population I stars. Further work is needed before a well accepted conclusion can be drawn.

\subsection{New observational results on the metal-poor tail of the Spite plateau}

Very few unevolved stars at extremely low metallicity are bright enough to be subjected to the spectroscopic study of the weak $\mathrm{Li}$ feature. A sudden drop in the ${ }^{7} \mathrm{Li}$ abundance at metallicities below 0.01 the solar metallicity is quite visible in a diagram plotted not in logarithmic coordinates, but on a linear scale (see Fig. 22 in Asplund et al. 2006 [15], referred to as ALN06 further on). In a study of the unevolved metal-poor halo stars, Bonifacio et al. 2007 [16] have studied 17 objects with metallicities between $-3.8<[\mathrm{Fe} / \mathrm{H}]<-2.7$ (see Fig. 1). This study supports the conclusion that in this metallicity range something is happening to the Spite plateau: (i) the scatter in $\mathrm{Li}$ abundance is much larger here than on the plateau $(-2.5<[\mathrm{Fe} / \mathrm{H}]<-1.5)$. The double-lined spectroscopic binary CS 22876-032 is a striking example of this behavior. Component A (higher mass) has a logarithmic ${ }^{7} \mathrm{Li}$ abundance of 2.2, component $\mathrm{B}$ has a Li abundance of only 1.75 ([17], see also Fig. 1). (ii) a decline of the abundance of ${ }^{7} \mathrm{Li}$ at very low metallicity looks probable. Garcia Pérez 2008 [18] also finds a drop at these lowest metallicities, which can be connected to the strict upper limit of ${ }^{7} \mathrm{Li}<0.6$ found in the ultra-metal-poor star HE 1327-2326 at $[\mathrm{Fe} / \mathrm{H}]=-5.3$ (Frebel et al. 2008 [19]). 


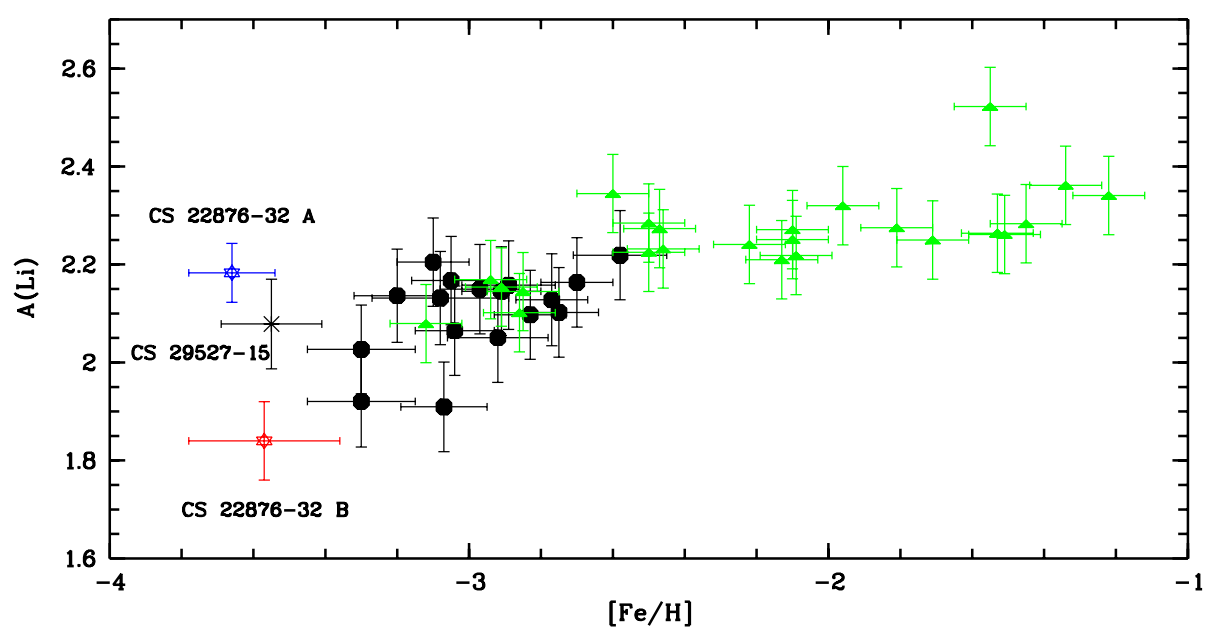

Figure 1: The metal-poor end of the Spite plateau. Note the increased scatter, and the trend towards a drop of the Li abundance at the lowest mean metallicities. Adapted from Bonifacio et al. 2007 [16] and González Hernández et al. 2008 [17].

\section{The ${ }^{6} \mathbf{L i}$ problem}

Until 2006, only very few determinations of the abundance of ${ }^{6} \mathrm{Li}$ were performed. The observation is very difficult, the resonance line of ${ }^{6} \mathrm{Li}$ being a weak doublet, blended with the corresponding doublet of ${ }^{7} \mathrm{Li}$, shifted by only $0.16 \AA$ to the red of the ${ }^{7} \mathrm{Li}$ line, and about 20 times weaker. The doublets are not resolved because of the thermal and turbulent broadening of the lines. So, the presence of ${ }^{6} \mathrm{Li}$ is detected only by a slight extra depression of the red wing of the unresolved ${ }^{7} \mathrm{Li}$ feature (Fig. 3). A drastic change occurred with the paper by Asplund et al. 2006 [15], who published a set of 24 determinations of ${ }^{6} \mathrm{Li}$ abundances, based on high quality spectra acquired with ESO's VLT/UVES combination. The surprising result was a kind of ${ }^{6} \mathrm{Li}$ plateau (top of Fig. 23 of their paper) at the level $\log n\left({ }^{6} \mathrm{Li}\right) \approx 0.8$. As clearly demonstrated by their Figure 23 this behavior does not fit the expectations based on cosmic ray (CR) production (again their Fig. 23, bottom). This result has immediately triggered new theoretical papers trying to explain the new ${ }^{6} \mathrm{Li}$ plateau.

\subsection{The pregalactic Cosmic Ray solution}

Rollinde, Vangioni \& Olive 2006 [20] have considered pregalactic cosmic rays generated by shocks around population III objects. By collisions between accelerated and at rest helium nuclei, the so called $\alpha$ fusion process generates both Lithium isotopes by the reactions ${ }^{4} \mathrm{He}(\alpha, \mathrm{D}){ }^{6} \mathrm{Li}$ and ${ }^{4} \mathrm{He}(\alpha, \mathrm{p})^{7} \mathrm{Li}$. This process works, but needs a very large fraction of the kinetic energy of population III SNe to be converted into CR energy, as noted by Prantzos 2006 [21] and Evoli et al. 2008 [22]. It seems also difficult to produce a plateau - in the diagram $\log n(6 \mathrm{Li})$ vs. $[\mathrm{Fe} / \mathrm{H}]-$ in the hierarchical model of Galaxy formation of Evoli et al. 2008. Other scenarios involving CR production have been proposed, but are exposed to similar critics.

\subsection{The supersymmetric particle solution}

See the list of references given in section 1.2, and paper by B. Fields (NIC-X, this volume). 

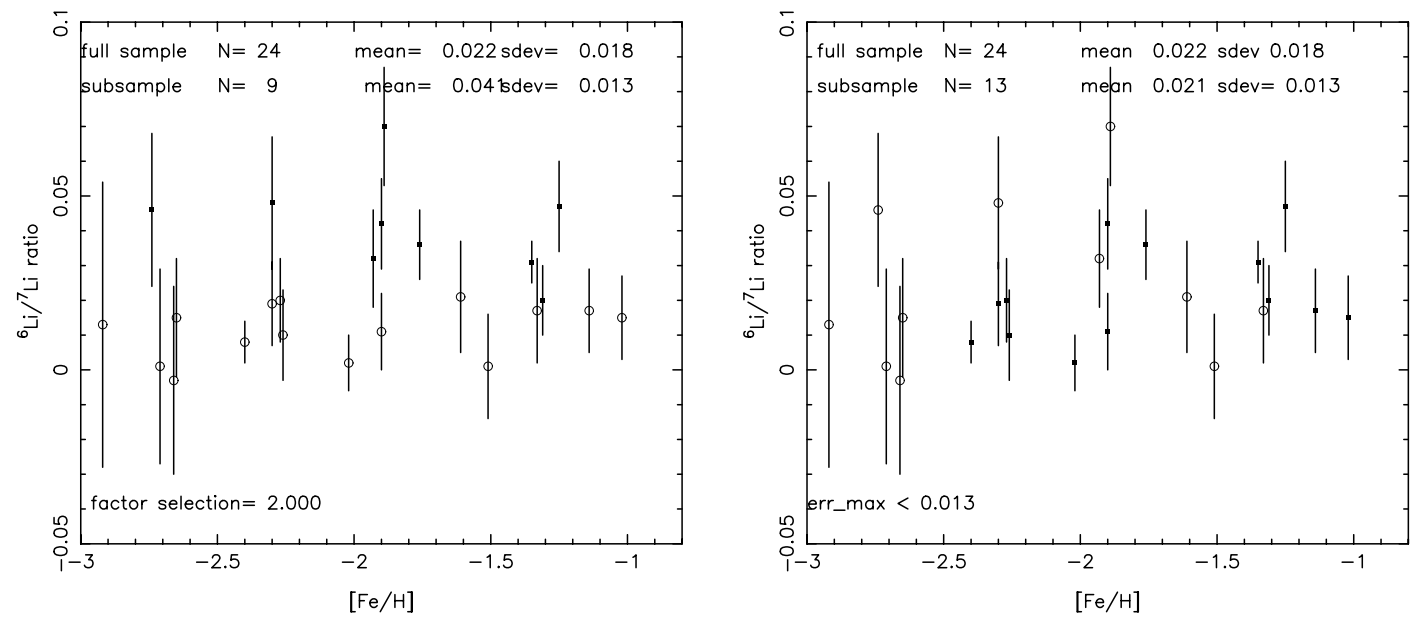

Figure 2: left panel:Fig. 13 (middle) of ALN06. Selected objects: filled dots, discarded objects: open circles. The mean value of $n\left({ }^{6} \mathrm{Li}\right) / n\left({ }^{7} \mathrm{Li}\right)$ of the full sample is 0.022 , the mean value of the selected subsample is 0.041. Right panel: Same as left panel excepted tha the selection is now done on the accuracy only error $<0.013$. The mean value is not affected now

\subsection{The ${ }^{7} \mathrm{Li}$ asymmetry solution: new results}

Observationally, the ${ }^{6} \mathrm{Li}$ measurement relies on the detection of a slight extra depression of the red wing of the ${ }^{7} \mathrm{Li}$ feature. However, such a line asymmetry already exists without the presence of ${ }^{6} \mathrm{Li}$. 3D hydrodynamical models of convection produced by the CO${ }^{5} \mathrm{BOLD}$ code [23] clearly predict a convection-induced line asymmetry of similar strength. Cayrel et al. 2007 [24] have shown that, in the case of the star HD74000, a halo turn-off star of metallicity $[\mathrm{Fe} / \mathrm{H}]=-2.0$, the derived ${ }^{6} \mathrm{Li}$ abundance is reduced to a negligible amount when taking into account this intrinsic line asymmetry. It was also shown that iron lines with equivalent widths similar to those of the doublet components of the ${ }^{7} \mathrm{Li}$ feature, and with the same stratification, show about the same amount of asymmetry as the computed one for ${ }^{7} \mathrm{Li}$. This is the only "sanity" check suggesting that our computed asymmetries are correct. The referee asked us if we could compare our asymmetries with those obtained with the 3D models used in ALN06, for example. This was not possible for ${ }^{7} \mathrm{Li}$, by lack of published data. We tried to use the published data in Allende Prieto et al. [25] on iron bisectors for Procyon. However in the short time available we do not claim to have reached a definite conclusion. Work is in progress to investigate this important point.

The big question is to know what amount of ${ }^{6} \mathrm{Li}$ would remain in the Asplund et al. analysis of 24 halo stars if the natural asymmetry of ${ }^{7} \mathrm{Li}$ had been properly included. In order to get some idea of that, we have extended the computation of the theoretical asymmetry of the ${ }^{7} \mathrm{Li}$ feature to other values of the parameters metallicity, effective temperature and gravity, spanning the range of Asplund et al. observations. Specifically, new computations have been performed for the central effective temperature of the sample, $T_{\mathrm{eff}}=6300 \mathrm{~K}$, at the 3 metallicities $[\mathrm{Fe} / \mathrm{H}]=-3.0,-2.0$, and -1.0 , and for the two gravities $\log g=4.0$ (turn-off) and 4.5 (unevolved dwarfs). At the central metalliciy, $[\mathrm{Fe} / \mathrm{H}]=-2.0$, the computations were made also for $T_{\text {eff }}=5900 \mathrm{~K}$ and again for $\log g=4.0$ and 4.5. The results of these computations are presented below. 


\subsubsection{A statistical problem with the subsample with detected ${ }^{6} \mathrm{Li}$}

Before proceeding further, it is worth to note that what everybody calls the ${ }^{6} \mathrm{Li}$ plateau is the almost constant ${ }^{6} \mathrm{Li}$ abundance of the stars having a certain detection of ${ }^{6} \mathrm{Li}$, namely those satisfying the condition: $\left({ }^{6} \mathrm{Li}\right) /\left({ }^{7} \mathrm{Li}\right)>2 \sigma ; \sigma$ being the estimated accuracy in the determination of this ratio. As the abundance of ${ }^{7} \mathrm{Li}$ is almost constant in the sample, and the noise is also fairly constant, it means that all values of $n\left({ }^{6} \mathrm{Li}\right) / n\left({ }^{7} \mathrm{Li}\right)$ below about 2 per cent have been discarded. This is clear in Fig. 13 of ALN06, reproduced here as Fig. 2. We are interested in the meaning of the "plateau" with respect to the full sample we note that the mean of the ratio for the full sample is 0.022 , compared to 0.041 for the subsample. The median of the full sample is 0.018 . A selection criterion involving the value of the studied variable is well known to introduce a severe bias (see the warning of Kapteyn \& Weersma 1910 [26] about small parallaxes). A selection with $n\left({ }^{6} \mathrm{Li}\right) / n\left({ }^{7} \mathrm{Li}\right)$ $>3 \sigma$ would move the plateau to a still higher value of 0.045 , whereas a selection on the error only, keeping errors below 0.013 , when the mean error is 0.0154 , leads to a mean of $n\left({ }^{6} \mathrm{Li}\right) / n\left({ }^{7} \mathrm{Li}\right)=0.021$, almost unchanged (Fig. 2). A more severe constraint on the accuracy of the determinations, error $<0.012$, gives a mean of 0.18 , still close to the mean of the full sample. In what follows we claim that the true measured signal is about 2 per cent induced by the asymmetry of ${ }^{6} \mathrm{Li}$ (affected by the measurement error). The mean of 0.022 acquires then a new signification: absence of ${ }^{6} \mathrm{Li}$. The certain detections are now decreased in number, as only values of $2 \sigma$ above 0.02 represent certain detections. The new number is of the order of 1 or 2 , and critically depends on the value of $\sigma$.

\subsubsection{The size and behavior of the convective line asymmetry}

Our main interest is investigate the dependence of the convective line asymmetry upon stellar metallicity, effective temperature and surface gravity. Table 1 gives our preliminary results for a small grid of 8 hydrodynamical simulations. The entry 'asym' is the excess of absorption in the red wing of the blend relative to the equivalent width of the full line. 'Asym' was determined by fitting the asymmetric 3D line profile, obtained from NLTE line formation calculations on a number $(N)$ of selected snapshots from the hydrodynamical simulations, with a combination of two symmetric $1 \mathrm{D}$ line profiles. It is what is measured in practice to determine the ratio $n\left({ }^{6} \mathrm{Li}\right) / n\left({ }^{7} \mathrm{Li}\right)$.

For given $T_{\text {eff }}$ and $\log g$, the predicted line asymmetry is fairly constant for metallicities below $[\mathrm{Fe} / \mathrm{H}]=-2$, but increases noticeably towards higher $[\mathrm{Fe} / \mathrm{H}]$. Hotter stars with lower gravity (turnoff stars) show higher line asymmetries than cooler unevolved dwarfs.

\subsection{The source of the line asymmetry}

It is interesting to have a look at the dynamical origin of the line asymmetry. Obviously, it is the hydrodynamical asymmetry between upward and downward convective flows that is at the origin of the asymmetry of the 3D line profiles. Fig. 5 shows a histogram of the vertical velocity at 4 optical depths in a typical 3D hydrodynamical model $\left(T_{\text {eff }}=6250 \mathrm{~K}, \log g=4.0,[\mathrm{Fe} / \mathrm{H}]=-2.0\right)$. There is little asymmetry at $\log \tau_{\text {ross }}=-3$ or -2 , but in the lower photosphere $\left(\log \tau_{\text {ross }}=-1\right.$ and 0 ) the asymmetry is conspicuous. For the centre of the disc, the 3D-NLTE contribution function of the $\mathrm{Li}$ I resonance line peaks at $\log \tau_{\text {ross }} \approx-1$. The broadening is large and the asymmetry is important (Fig. 5). In 3D-LTE, however, the contribution function has a double peak, one at $\log \tau_{\text {ross }} \approx-1$, but a second one at in very superficial and very cool layers [24], where the velocity distribution is 


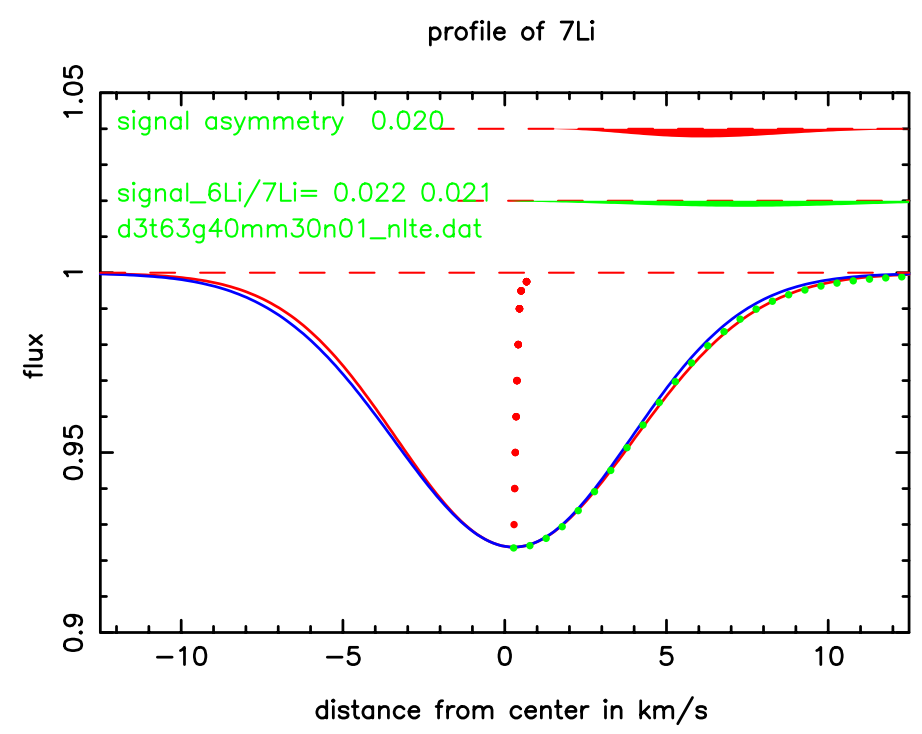

Figure 3: The red line is a (intrinsically asymmetric) 3D-NLTE profile of the major component of ${ }^{7} \mathrm{Li}$ (area $W$ ), computed for atmospheric parameters $T_{\text {eff }}=6250 \mathrm{~K}, \log g=4.0,[\mathrm{Fe} / \mathrm{H}]=-3.0$. The blue line is the mirror image (with respect to the bisector of the line core) of the red line completing a symmetric profile with the left wing of the red profile. The difference between the right wings of the red and the blue profiles (area $\Delta W$ ) measures the amount of asymmetry of the 3D profile ( The green dots represent the profile of a ${ }^{6} \mathrm{Li} /{ }^{7} \mathrm{Li}$ blend, computed with the symmetric profile and a ratio $n\left({ }^{6} \mathrm{Li}\right) / n\left({ }^{7} \mathrm{Li}\right)=0.022$, equal to the mean value of the observations of ALN06 for a selection of stars with parameters $6100 \mathrm{~K}<T_{\text {eff }}<6400 \mathrm{~K}$ and $3.7<\log g<4.3$. The signal of this blend measures the isotopic ratio: $n\left({ }^{6} \mathrm{Li}\right) / n\left({ }^{7} \mathrm{Li}\right)=\Delta W / W=0.021$. There is a slight difference between the signals produced by the asymmetric $3 \mathrm{D}$ profile and the ${ }^{6} \mathrm{Li} /{ }^{7} \mathrm{Li}$ blend (the ${ }^{6} \mathrm{Li}$ blend is slightly more red-shifted and shallower), but these differences are too small to be measurable.

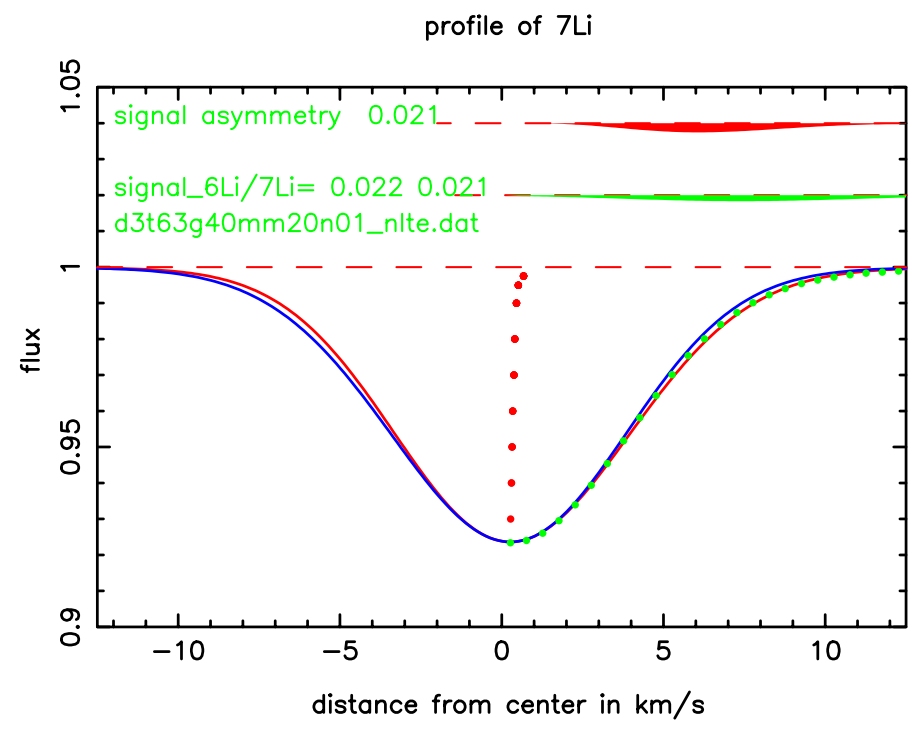

Figure 4: Same as fig 3, except that here $[\mathrm{Fe} / \mathrm{H}]=-2.0$. 


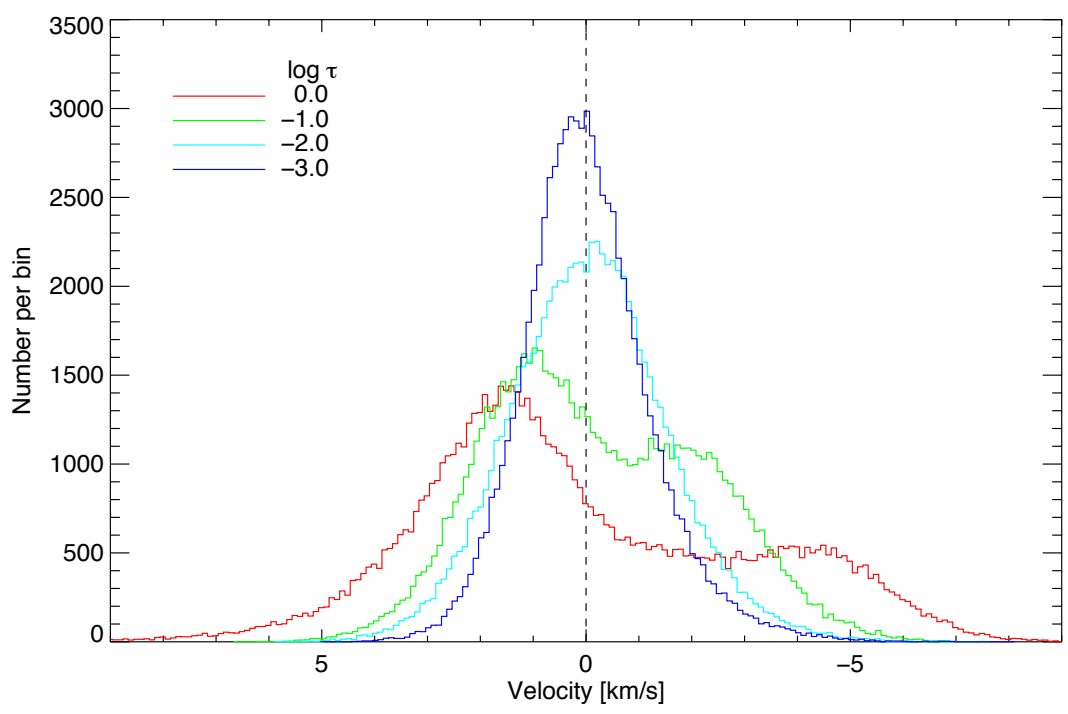

Figure 5: Histogram of vertical velocities at 4 optical depths in the atmosphere of a typical metal-poor star $\left(T_{\text {eff }}=6250 \mathrm{~K}, \log g=4.0,[\mathrm{Fe} / \mathrm{H}]=-2.0\right)$. Positive (negative) velocities correspond to blue- (red-) shifts. See text for details

\begin{tabular}{llllll}
\hline$T_{\text {eff }}$ & $\log g$ & {$[\mathrm{Fe} / \mathrm{H}]$} & asym. & $N$ & $X \times Y \times Z\left[\mathrm{Mm}^{3}\right]$ \\
\hline 6250 & 4.0 & -3.0 & 0.020 & 20 & $26 \times 26 \times 12.7$ \\
6250 & 4.0 & -2.0 & 0.021 & 16 & $26 \times 26 \times 12.7$ \\
6250 & 4.0 & -1.0 & 0.037 & 20 & $26 \times 26 \times 12.7$ \\
6250 & 4.5 & -3.0 & 0.012 & 18 & $7.0 \times 7.0 \times 3.9$ \\
6250 & 4.5 & -2.0 & 0.011 & 19 & $7.0 \times 7.0 \times 3.9$ \\
6250 & 4.5 & -1.0 & 0.017 & 20 & $7.0 \times 7.0 \times 3.9$ \\
\hline 5900 & 4.0 & -2.0 & 0.015 & 20 & $26 \times 26 \times 12.5$ \\
5900 & 4.5 & -2.0 & 0.011 & 19 & $6.0 \times 6.0 \times 3.8$
\end{tabular}

Table 1: Computed line asymmetry expressed as equivalent isotopic ratio ${ }^{6} \mathrm{Li} /{ }^{7} \mathrm{Li}$ (asym.), and $3 \mathrm{D}$ model properties: $T_{\text {eff }}$ is the effective temperature, $\log g$ the surface gravity, $[\mathrm{Fe} / \mathrm{H}]$ the metallicity, $N$ the number of considered statistically independent snapshots, and $X \times Y \times Z$ the geometrical extend of the computational box. All models use six wavelength bins to represent the wavelength dependence of the radiative energy exchange, and a grid of $140 \times 140 \times 150$ cells.

narrower and virtually symmetric. This explains why the 3D-LTE analysis of ALN06 differs from our 3D-NLTE analysis. There is a substantial difference in the two profiles, the 3D-LTE profile being a superposition of an asymmetric line (the part formed in the lower photosphere) and of a symmetric line (with a different shift) formed in the higher photospheric layers.

Clearly, the real situation is more complex than suggested by the simplistic picture outlined above. It is not only the distribution of the vertical velocity but also its correlation with the temperature fluctuations that determines the shape of the line profile. Moreover, horizontal motions also become important when integrating the line profile across the stellar disk. 


\subsection{Conclusion}

The level of the plateau attributed to ${ }^{6} \mathrm{Li}$ in ALN06 [15] is affected by a bias which, when corrected, moves the plateau to a mean value of ${ }^{6} \mathrm{Li} /{ }^{7} \mathrm{Li} \approx 0.022$. The study of the ${ }^{7} \mathrm{Li}$ line asymmetry with a 3D-NLTE code on 3D hydrodynamical models obtained with the CO5 BOLD code leads to asymmetry signals of respectively $0.020,0.021$ for $[\mathrm{Fe} / \mathrm{H}]=-3.0$ and -2.0 respectively, at $T_{\mathrm{ff}}=$ $6250 \mathrm{~K}, \log g=4.0$. Stars corresponding to the subsample of ALN06 with $6100 \mathrm{~K}<T_{\text {eff }}<6400 \mathrm{~K}$, $3.7<\log g<4.3$, have a ratio $n\left({ }^{6} \mathrm{Li}\right) / n\left({ }^{7} \mathrm{Li}\right)$ with a mean value of 0.021 (14 objects). For metallicity $[\mathrm{Fe} / \mathrm{H}]=-1$, the predicted asymmetry signal is 0.037 , and the observed value in ALN06 is 0.023 , not a very good fit but the number of objects is only 4 . The effect of the gravity is a decrease of the asymmetry by a factor of 2 when $\log g=4.5$ instead of 4.0. The only certain unevolved star in the ALN06 sample is HD19445: observed signal: 0.002, asymmetry predicted 0.010. The conclusion is that the plateau of the remarkable ALN06 set of observations can be reinterpreted without a contribution by ${ }^{6} \mathrm{Li}$. However, a detailed re-analysis of a few stars with the highest apparent ${ }^{6} \mathrm{Li}$ content is necessary before a final conclusion can be drawn for them.

\section{References}

[1] Spergel, D. N., R. Bean, O. Doré, et al., Three-Year Wilkinson Microwave Anisotropy Probe (WMAP) Observations: Implications for Cosmology, ApJS, 170,377 (2007)

[2] Charbonnel, C. \& F. Primas, The lithium content of the Galactic Halo stars, A\&A, 442,961 (2005)

[3] Piau, L., T. C. Beers, D. S. Balsara, T. Sivarani, J. W. Truran, \& J. W. Ferguson, From First Stars to the Spite Plateau: A Possible Reconciliation of Halo Stars Observations with Predictions from Big Bang Nucleosynthesis, ApJ, 653,300 (2006)

[4] N., Prantzos Early galactic chemical evolution, Proceedings of the International Symposium on Nuclear Astrophysics - Nuclei in the Cosmos - IX. 25-30 June 2006, CERN, PoS p.254 , (2006)

[5] Jedamzik, K., Neutralinos, big bang nucleosynthesis, and ${ }^{6}$ Li in low-metallicity stars, PhRvD, 70,083510 (2004)

[6] Jedamzik, K., Cosmic Li6 and Li7 problems and BBN with long-lived charged massive particles, PhRvD, 77,063524 (2008)

[7] Pospelov, M., Bridging the primordial A=8 divide with Catalyzed Big Bang Nucleosynthesis, arXiv:0712.0647 (2007)

[8] Cumberbatch, D., K. Ichikawa, M. Kawasaki, K. Kohri, J. Silk, \& G. D. Starkman, Solving the cosmic lithium problems with primordial late-decaying particles, $\mathrm{PhRvD}, \mathbf{7 6}, 123005$ (2007)

[9] Kusakabe, M., T. Kajino, R. N. Boyd, T. Yoshida, \& G. J. Mathews, Simultaneous solution to the Li6 and Li7 big bang nucleosynthesis problems from a long-lived negatively charged leptonic particle, $\mathrm{PhRvD}, \mathbf{7 6}, 121302$ (2007)

[10] Alecian, G., O. Richard, \& S. Vauclair, Element stratification in stars : 40 years of atomic diffusion; Meeting in Honour of Georges Michaud, EAS, 17,(2005)

[11] Korn, A. J., F. Grundahl, O. Richard, L. Mashonkina, P. S. Barklem, R. Collet, B. Gustafsson, \& N. Piskunov, Atomic Diffusion and Mixing in Old Stars. I. Very Large Telescope FLAMES-UVES Observations of Stars in NGC 6397, ApJ, 671,402 (2007) 
[12] Boesgaard, A. M. \& M. J. Tripicco, Lithium in the Hyades Cluster, ApJ, 302,L49 (1986)

[13] Boesgaard, A. M., C. P. Deliyannis, A. Stephens, \& D. L. Lambert, Boron in Lithium-and Beryllium-deficient F Stars, ApJ, 492,727 (1998)

[14] Charbonnel, C. \& S. Talon, Deep inside low-mass stars, IAUS, 252,163 (2008)

[15] Asplund, M., D. L. Lambert, P. E. Nissen, F. Primas, \& V. V. Smith, Lithium Isotopic Abundances in Metal-poor Halo Stars, ApJ, 644,229 (2006)

[16] Bonifacio, P., P. Molaro, T. Sivarani, et al., First stars VII - Lithium in extremely metal poor dwarfs, A\&A, 462,851 (2007)

[17] González Hernández, J. I., P. Bonifacio, H.-G. Ludwig, et al., First stars XI. Chemical composition of the extremely metal-poor dwarfs in the binary CS 22876-032, A\&A, 480,233 (2008)

[18] Garcia Perez, A.E. Poster at this meeting (2008)

[19] Frebel, A., R. Collet, K. Eriksson, N. Christlieb, \& W. Aoki, HE 1327-2326, an Unevolved Star with $[\mathrm{Fe} / \mathrm{H}]<-5.0$. II. New 3D-1D Corrected Abundances from a Very Large Telescope UVES Spectrum, ApJ, 684,588 (2008)

[20] Rollinde, E., E. Vangioni, \& K. A. Olive, Population III Generated Cosmic Rays and the Production of ${ }^{6} \mathrm{Li}, \mathrm{ApJ}, \mathbf{6 5 1}, 658$ (2006)

[21] Prantzos, N., The energetics, evolution, and stellar depletion of ${ }^{6} \mathrm{Li}$ in the early Galaxy, A\&A, 448,665 (2006)

[22] Evoli, C., S. Salvadori, \& A. Ferrara, The puzzling origin of the ${ }^{6}$ Li plateau, MNRAS, 390,L14 (2008)

[23] Wedemeyer, S., B. Freytag, M. Steffen, H.-G. Ludwig, \& H. Holweger, Numerical simulation of the $3 D$ structure and dynamics of the non-magnetic solar chromosphere, A\&A, 414,1121 (2004)

[24] Cayrel, R., M. Steffen, H. Chand, et al., Line shift, line asymmetry, and the ${ }^{6} \mathrm{Li} /{ }^{7} \mathrm{Li}$ isotopic ratio determination, A\&A, 473,L37 (2007)

[25] C. Allende Prieto, M. Aplund,G., R.J. García López,Signature of convection in the spectrum of Procyon: fundamental parameters and iron abundance, Astrophys.J. 567, 544 (2002)

[26] Kapteyn, J. C. \& H. A. Weersma, List of parallax determinations compiled by Prof. J. C. Kapteyn and Dr. H. A. Weersma, PGro, 24,1 (1910) 